\title{
Perceptually based parameter adjustments for video processing operations
}

\author{
Gabriel Eilertsen $^{1} \quad$ Jonas Unger ${ }^{1}$ \\ Robert Wanat ${ }^{2}$ \\ Rafał Mantiuk $^{2}$ \\ ${ }^{1}$ Linköping University, Sweden \\ ${ }^{2}$ Bangor University, United Kingdom
}
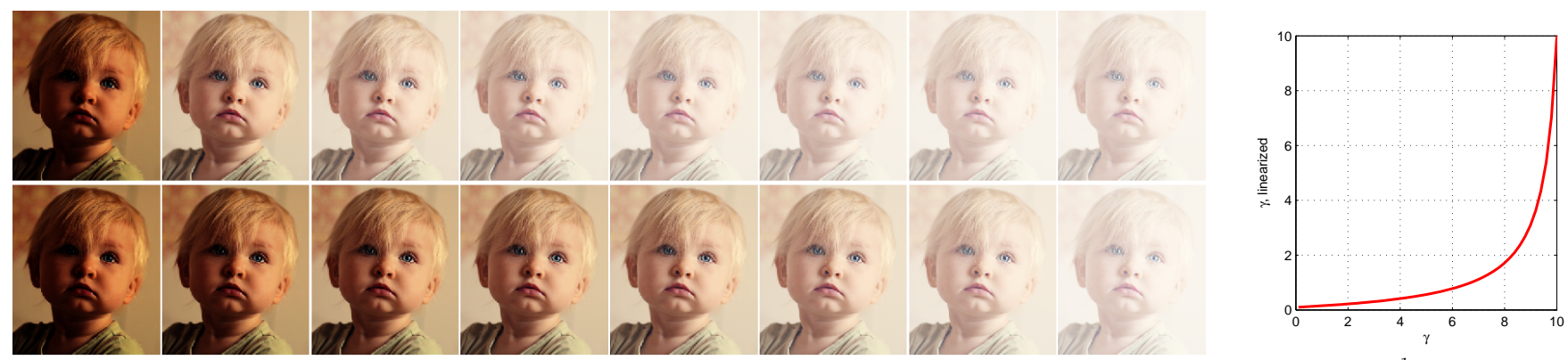

Figure 1: Example linearization, for demonstration purpose, using a simple gamma mapping value as parameter, $\boldsymbol{I}_{p}=\boldsymbol{I}^{\frac{1}{p}}$. Left: Output with equal sized steps of gamma (top) and after linearization (bottom). Right: The transformation function, $\hat{f}^{-1}(p)$.

\section{Introduction}

Extensive post processing plays a central role in modern video production pipelines. A problem in this context is that many filters and processing operators are very sensitive to parameter settings and that the filter responses in most cases are highly non-linear as illustrated in Figure 1 (left). Since there is no general solution for performing perceptual calibration of image and video operators automatically, it is often necessary to manually perform tweaking of multiple parameters. This is an iterative process which requires instant visual feedback of the result in both the spatial and temporal domains. Due to large filter kernels, computational complexity, high frame rate, and image resolution it is, however, often very time consuming to iteratively re-process and tweak long video sequences.

We present a new method for rapidly finding the perceptual minima in high-dimensional parameter spaces of general video operators. The key idea of our algorithm is that the characteristics of an operator can be accurately described by interpolating between a small set of pre-computed parameter settings. By computing a perceptual linearization of the parameter space of a video operator, the user can explore this interpolated space to find the best set of parameters in a robust way. Since many operators are dependent on two or more parameters, we formulate this as a general optimization problem where we let the objective function be determined by the user's image assessments. To demonstrate the usefulness of our approach we show a set of use cases (see the supplementary material) where our algorithm is applied to computationally expensive video operations.

\section{Parameter space linearization}

The goal of the linearization is to determine the transformation of each parameter to a perceptually linear domain, see Figure 1. This could be achieved experimentally by performing a perceptual comparison study [Wills et al. 2009]. However, to be able to find this transformation automatically for any operator and parameter, we suggest analyzing changes in the image output. As a simple and robust measure we use the absolute difference summed over the pixels, and require it to change linearly for each parameter. We formulate the image change function as in Equation 1, where $\boldsymbol{I}_{p}$ denotes the image $\boldsymbol{I}$ processed with parameter $p$, and use the normalized inverse function $\hat{f}^{-1}$ as a parameter transformation. We have found that the result of this mapping corresponds closely to a perceptually linear space for most parameters (see supplementary material).

$$
f(p)=\int\left(\sum\left|\frac{d \boldsymbol{I}_{p}}{d p}\right|\right) d p
$$

Please note that this is a pre-computation step that can be computed on one or more video sequences and that the resulting linearization transformation can be used across different video sequences.

\section{Interpolated parameter adjustment}

The linearization allows us to accurately describe and explore the full parameter space using only a sparse set of sampling points (typically 3-4 pre-computed parameter settings along each search direction). To find a perceptual minima of this space we search one direction at the time, choosing directions according to Powell's conjugate gradient method [Powell 1964], as explained in Algorithm 1. The method allows for finding a minima of a non-differentiable objective function (the user) in a high-dimensional parameter space. The iterative nature of our approach makes it robust against errors. If inaccuracies are introduced by the user the solution will converge more slowly. However, performing additional iterations will ensure convergence towards a perceptual minima.

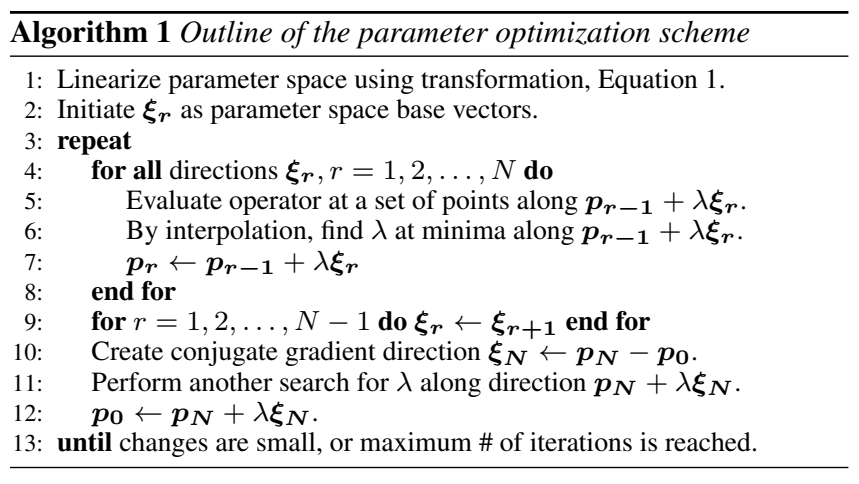

\section{Evaluation and results}

To evaluate our method, we applied it to calibration of video Tone Mapping Operators (TMOs) [Eilertsen et al. 2013]. The linearizations were, for each TMO, calculated as the average over several video sequences. The optimization used five sample points in the parameter spaces and was performed with four observers. The final parameters for the TMOs were obtained as the average from the four observers and showed good results when applied to the different HDR video clips in the study (see the supplementary material).

\section{References}

Eilertsen, G., Wanat, R., Mantiuk, R. K., AND Unger, J. 2013. Evaluation of tone mapping operators for hdr-video. Computer Graphics Forum 32, 7, 275-284.

Powell, M. J. D. 1964. An efficient method for finding the minimum of a function of several variables without calculating derivatives. The Computer Journal 7, 2 (Jan.).

Wills, J., Agarwal, S., Kriegman, D., and Belongie, S. 2009. Toward a perceptual space for gloss. ACM Trans. on Graph. 28, 4 (Sept.). 